\title{
15. DIFFUSION OF METEOR TRAINS IN THE GEOMAGNETIC FIELD
}

\author{
T. R. KAISER \\ (University of Sheffield, England)
}

\section{A BSTRACT}

It is shown that the Earth's magnetic field can have significant effects on diffusion only above about $95 \mathrm{~km}$ altitude. It is further predicted that diffusion can be markedly inhibited by the field only if the meteor train axis makes an angle of less than $1^{\circ}$ with the field direction. Experimental support for this view is presented.

\section{Introduction}

In the absence of a magnetic field, the ambipolar diffusion of a weakly ionized gas obeys a conventional diffusion equation of the form

$$
\frac{\partial N}{\partial t}=D \nabla^{2} N
$$

where quasi-neutrality is assumed, i.e. $N=N_{-}=n N_{+}$is the electron density with $n$-fold ionization. $D$ is the ambipolar diffusion coefficient, which is related to the positive ion and electron diffusion coefficients $D_{+}$and $D_{-}$, and mobilities $K_{+}$and $K_{-}$by

$$
D=\begin{gathered}
K_{-} D_{+}+K_{+} D_{-} \\
K_{-}+K_{+}
\end{gathered}
$$

Now $K_{-} / D_{-}=e\left(k T_{-}\right)^{-1}$ and $K_{+} / D_{+}=n e\left(k T_{+}\right)^{-1}$ where $T_{-}$and $T_{+}$are the electron and ion temperatures, $k$ is Boltzmann's constant and $e$ is the electronic charge. Hence

$$
D=\frac{D_{+}\left(1+n T_{-} / T_{+}\right)}{1+n \rho},
$$

where

$$
\rho=\frac{T_{-}}{T_{+}} \frac{D_{+}}{D_{-}}
$$

The mass ratio between ions and electrons ensures that $\rho \ll 1$, giving

$$
D \simeq D_{+}\left(1+n T_{-} / T_{+}\right)
$$

Kresák and Millman (eds.), Physics and Dynamics of Meteors, 161-168. O I.A.U. 
thus, if $n=1, T_{-}=T_{+}$, we get

$$
D \simeq 2 D_{+} .
$$

$D$ has been evaluated as a function of altitude and is plotted in Figure 1.

If we regard a meteor train as a line source which diffuses into a neutral atmosphere, the solution to Equation (1) is

$$
N=\frac{\alpha}{4 \pi D t} \exp \left(-r^{2} / 4 D t\right)
$$

where $\alpha=$ electron line-density, $t=$ time lapse after passage of the meteor, and $r=$ radius from the train axis.

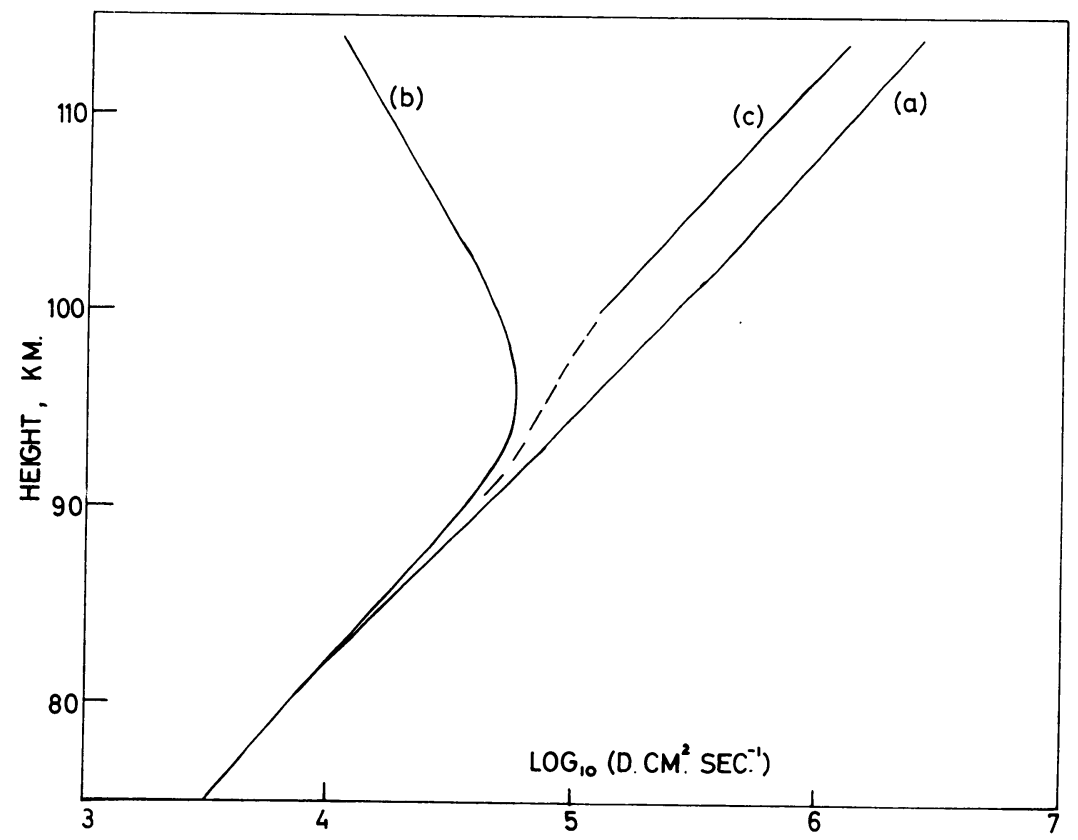

FIG. 1. (a) $2 D_{+}=$diffusion coefficient in absence of a magnetic field. - (b) $D_{T}$ as given by Equation (17) is the coefficient for transverse diffusion of a field-aligned meteor train. - (c) $D_{+}$, suggested as an upper limit to transverse diffusion at altitudes above $100 \mathrm{~km}$ or so.

The effect of a finite initial radius may be introduced by assuming the train to be created at time $t_{0} \neq 0$.

A magnetic field reduces the ionic and electron diffusion coefficients transverse to the field to $D_{T+}$ and $D_{T-}$, while leaving the longitudinal coefficients unchanged (equal to $D_{+}$and $D_{-}$), and it can be shown that

$$
D_{T \pm}=\frac{v_{ \pm}^{2}}{v_{ \pm}^{2}} \frac{\omega_{ \pm}^{2}}{ \pm} D_{ \pm}
$$


where $v_{+}, v_{-}$are the ion and electron collision frequencies and $\omega_{+}, \omega_{-}$are the gyro-frequencies. At meteor heights, $D_{T+}$ will not be very different from $D_{+}$, but $D_{T-}$ is greatly reduced at altitudes above about $95 \mathrm{~km}$. It is tempting to assume that the ambipolar diffusion will obey an anisotropic diffusion equation with longitudinal coefficient equal to $D$ and transverse coefficient $D_{T}<D$, i.e.

$$
\frac{\partial N}{\partial t}=D_{T}\left(\frac{\partial^{2} N}{\partial u^{2}}+\frac{\partial^{2} N}{\partial v^{2}}\right)+D \frac{\partial^{2} N}{\partial w^{2}},
$$

where $u, v, w$ are cartesian coordinates with the magnetic field in the $w$-direction.

For a line source at angle $\theta$ to the magnetic field, it is convenient to use coordinates $x, y, z$ where the $z$-axis is parallel to the source and the magnetic field is in the $(x, z)$ plane. In this case $\partial / \partial z=0$ and (8) becomes

$$
\frac{\partial N}{\partial t}=D_{x} \frac{\partial^{2} N}{\partial x^{2}}+D_{y} \frac{\partial^{2} N}{\partial y^{2}},
$$

where $D_{x}=D_{T} \cos ^{2} \theta+D \sin ^{2} \theta$ and $D_{y}=D_{T}$. The solution is now

$$
N=\frac{\alpha}{4 \pi t \sqrt{D_{x} D_{y}}} \exp \left[-\left(\frac{x^{2}}{4 D_{x} \mathrm{t}}+\frac{y^{2}}{4 D_{y} t}\right)\right] .
$$

It is not difficult to show that the echo from an underdense meteor train $\left(\alpha \lesssim 10^{12} \mathrm{~cm}^{-1}\right.$ ), with electron density given by (10), will decay exponentially with time constant $\tau$ given by

$$
\tau=\lambda^{2} /\left(16 \pi^{2} D_{\mathrm{eff}}\right)
$$

where $\lambda$ is the wavelength and the effective diffusion coefficient is given by

$$
D_{\text {eff }}=D_{x} \sin ^{2} \phi+D_{y} \cos ^{2} \phi \text {. }
$$

The incident ray is in the $x, y$ plane at angle $\phi$ to the $y$-axis. If $\psi$ is the angle between the incident specular ray (which is normal to the train axis) and the magnetic field, then $\cos \psi=\sin \theta \sin \phi$, hence we get the simple relation

$$
D_{\text {eff }}=D \cos ^{2} \psi+D_{T} \sin ^{2} \psi
$$

While the above seems very satisfactory, it will be shown that, in the presence of the magnetic field, we cannot describe the diffusion by a simple equation such as (8) and that the nature of the diffusion depends upon the initial conditions (geometry of the electron-ion cloud), i.e., for the meteor case, on the angle $\theta$ to the magnetic field.

\section{Diffusion in a Magnetic Field}

In addition to the coefficients $D_{T \pm}$ we must introduce the Hall coefficients $D_{\mathrm{H} \pm}$ 
given by

$$
D_{\mathrm{H} \pm}=\frac{v_{ \pm} \omega_{ \pm}}{v_{ \pm}^{2}+\omega_{ \pm}^{2}}
$$

and the transverse and Hall mobilities given by

$$
K_{T+} / D_{T+}=K_{\mathbf{H}_{+}} / D_{\mathrm{H}_{+}}=n e\left(k T_{+}\right)^{-1}, \quad K_{T-} / D_{T-}=K_{\mathrm{H}-} / D_{\mathrm{H}_{-}}=e(k T)^{-1} .
$$

The ion and electron diffusion equations now become

$$
\begin{aligned}
\frac{\partial N_{+}}{\partial t}=\frac{\partial}{\partial u}\left(D_{T+} \frac{\partial N_{+}}{\partial u}-\right. & \left.K_{T+} N_{+} E_{u}\right)+\frac{\partial}{\partial v}\left(D_{T+} \frac{\partial N_{+}}{\partial v}-K_{T+} N_{+} E_{v}\right) \\
& +\frac{\partial}{\partial w}\left(D_{+} \frac{\partial N_{+}}{\partial w}-K_{+} N_{+} E_{w}\right)-K_{\mathrm{H}+}\left[\nabla \wedge\left(N_{+} \mathbf{E}\right)\right]_{w}, \\
\frac{\partial N_{-}}{\partial t}=\frac{\partial}{\partial u}\left(D_{T-} \frac{\partial N_{-}}{\partial u}+\right. & \left.K_{T-} N_{-} E_{u}\right)+\frac{\partial}{\partial v}\left(D_{T-} \frac{\partial N_{-}}{\partial v}+K_{T-} N_{-} E_{v}\right) \\
& +\frac{\partial}{\partial w}\left(D_{-} \frac{\partial N_{-}}{\partial w}+K_{-} N_{-} E_{w}\right)-K_{\mathrm{H}-}\left[\nabla \wedge\left(N_{-} \mathbf{E}\right)\right]_{w},
\end{aligned}
$$

where $\mathbf{E}$ is the space charge electric field. In the absence of a magnetic field, the Hall terms are zero and $D_{T+}=D_{+}, D_{T-}=D_{-}$etc. in which case, assuming quasineutrality, the space charge field is easily eliminated leading to Equation (1). In the presence of a field the situation is much more complicated, partly because the particle flow is no longer wholly across the surfaces of constant density and differential flow can occur along these surfaces without upsetting quasi-neutrality.

It is of interest to see whether there is an initial configuration for which the Hall terms remain zero and for which quasi-neutrality requires the corresponding terms in (15) and (14) to have ratio $n$. In this case we find

$$
\begin{aligned}
E_{u} & =\frac{D_{T+}-D_{T-}}{K_{T+}+K_{T-}} \frac{1}{N} \frac{\partial N}{\partial u}, \\
E_{v} & =\frac{D_{T+}-D_{T-}}{K_{T+}+K_{T-}} \frac{1}{N} \frac{\partial N}{\partial v}, \\
E_{w}= & \frac{D_{+}-D_{-}}{K_{+}+K_{-}} \frac{1}{N} \frac{\partial N}{\partial w},
\end{aligned}
$$

and $\partial N / \partial t$ satisfies (8) with

$$
D_{T}=\frac{K_{T-} D_{T+}+K_{T+} D_{T-}}{K_{T-}+K_{T+}} .
$$

At altitudes below $95 \mathrm{~km}$, this becomes $D_{T} \simeq 2 D_{T+}$, i.e. the field has little effect; at greater altitudes we obtain $D_{T} \simeq 2 D_{T}$ - which is small compared with $D$ and decreases 
with increasing altitude. $D_{T}$, as given by (17), is plotted as a function of altitude in Figure 1. This result would imply that transverse diffusion is electron controlled at heights above $95 \mathrm{~km}$.

We can see straight away that the above result cannot generally be true since, although our approximation relates to a quasi-static space charge field, $\nabla \wedge \mathbf{E}$ obtained from Equation (16) is in general non-zero (although the field given by (16) satisfies the requirement that the Hall terms in (14) and (15) are zero). It can be shown that $\nabla \wedge \mathbf{E}=0$ if $N(u, v, w)$ can be written as a product of separate functions $N=U(u) V(v) W(w)$, however this leads to physically unrealizable forms for the space-charge field. Thus the only remaining case satisfying $\nabla \wedge \mathbf{E}=0$ is that for which $\partial / \partial w=0$, i.e. $E_{w}=0$. In other words, only if the meteor train is aligned with the magnetic field will the transverse diffusion coefficient be given by Equation (17).

\section{Some Physical Considerations}

The above results can be explained physically as follows. In the absence of a magnetic field, $D_{-} \gg D_{+}$, hence the electrons tend to diffuse more rapidly than the ions and a positive space charge forms. The resulting electric field is such as to inhibit charge separation and the diffusion is ion-controlled. If the ionization is aligned with the magnetic field, and if $D_{T-} \ll D_{T+}$, then the ions tend to escape from the electrons, the space charge is negative and the diffusion is controlled by the electrons. For the more general case of an ion cloud in a magnetic field, the effective transverse ambipolar diffusion will depend upon the nature of the space charge created. Since the electron mobility along the field lines is extremely large compared with that of the ions, the space charge will generally be positive unless the density gradient along the field is very small compared with that transverse to the field. Thus, for a meteor train, we might expect the effective transverse diffusion coefficient to be given by (17) only if the angle between the train and the field is small enough - the question is how small? At larger angles the inhibition of diffusion across the field will be greatly reduced.

\section{A Special Case}

While it has not been possible to obtain a solution satisfying (14) and (15) for a line source at an angle to the field, a result obtained for the case of a plane slab of ionization at angle $\theta$ to the field is particularly illuminating. If the $v$-axis lies in the plane of the slab, then the diffusion can be shown to obey the equation

$$
\frac{\partial N}{\partial t}=D_{T} \frac{\partial^{2} N}{\partial u^{2}}+D_{L} \frac{\partial^{2} N}{\partial w^{2}},
$$


where

and

$$
D_{T}=\frac{2 D_{T+} D_{T-} \cos ^{2} \theta+\left(D_{T+} D_{-}+D_{T-} D_{+}\right) \sin ^{2} \theta}{\left(D_{T-}+D_{T+}\right) \cos ^{2} \theta+\left(D_{-}+D_{+}\right) \sin ^{2} \theta}
$$

$$
D_{L}=\frac{\left(D_{+} D_{T-}+D_{-} D_{T+}\right) \cos ^{2} \theta+2 D_{+} D_{-} \sin ^{2} \theta}{\left(D_{T-}+D_{T+}\right) \cos ^{2} \theta+\left(D_{-}+D_{+}\right) \sin ^{2} \theta}
$$

are the transverse and longitudinal coefficients. The important feature of this result is that it shows $D_{T}$ and $D_{L}$ to be functions of $\theta$, i.e. of the configuration of the ion-cloud relative to the field. In the absence of a field, and for $\theta=0$, Equations (19) and (20) reduce to the results previously obtained.

If we introduce a new coordinate, $x$, measured in the direction normal to the planes of constant density, Equation (18) simplifies to

where

$$
\frac{\partial N}{\partial t}=D(\theta) \frac{\partial^{2} N}{\partial x^{2}}
$$

$$
D(\theta)=\frac{2\left(D_{T-} \cos ^{2} \theta+D_{-} \sin ^{2} \theta\right)\left(D_{T+} \cos ^{2} \theta+D_{+} \sin ^{2} \theta\right)}{\left(D_{T-}+D_{T+}\right) \cos ^{2} \theta+\left(D_{-}+D_{+}\right) \sin ^{2} \theta}
$$

This is plotted as a function of $\theta$, for various altitudes, in Figure 2 . Note that $D\left(90^{\circ}\right) \simeq 2 D_{+}=$value in the absence of a magnetic field.

\section{Discussion}

It is clear from Figure 2 that, at meteor heights, the magnetic field significantly inhibits the diffusion of a plane slab only if the angle $\theta$ is less than about $1^{\circ}$. The meteor train case is more complicated (e.g. the Hall terms in general will be non-zero) but, from the physical considerations discussed above, we would expect the result to be similar, i.e. only if the train is aligned with the magnetic field to within less than $1^{\circ}$ or so is any large reduction in diffusion likely. We might thus expect the diffusion in the plane containing the train axis and the field direction to vary somewhat as in Figure 2. When the angle to the magnetic field is appreciable, Equation (19) gives $D_{T} \simeq D_{T+} \simeq D_{+}$at altitudes much above $95 \mathrm{~km}$ (and of course $D_{T} \simeq 2 D_{+}$at lower altitudes). This suggests that, at these greater heights, $D_{+}$may be regarded possibly as the upper limit for diffusion transverse to the plane containing the train and the field direction.

Various workers who have measured the effective diffusion coefficient as a function of altitude, find that the diffusion scale height is in excess of the atmospheric density scale height. The ideas discussed here would suggest that the effective coefficient will be $2 D_{+}$below $95 \mathrm{~km}$ and generally a smaller value above, but nevertheless one which 


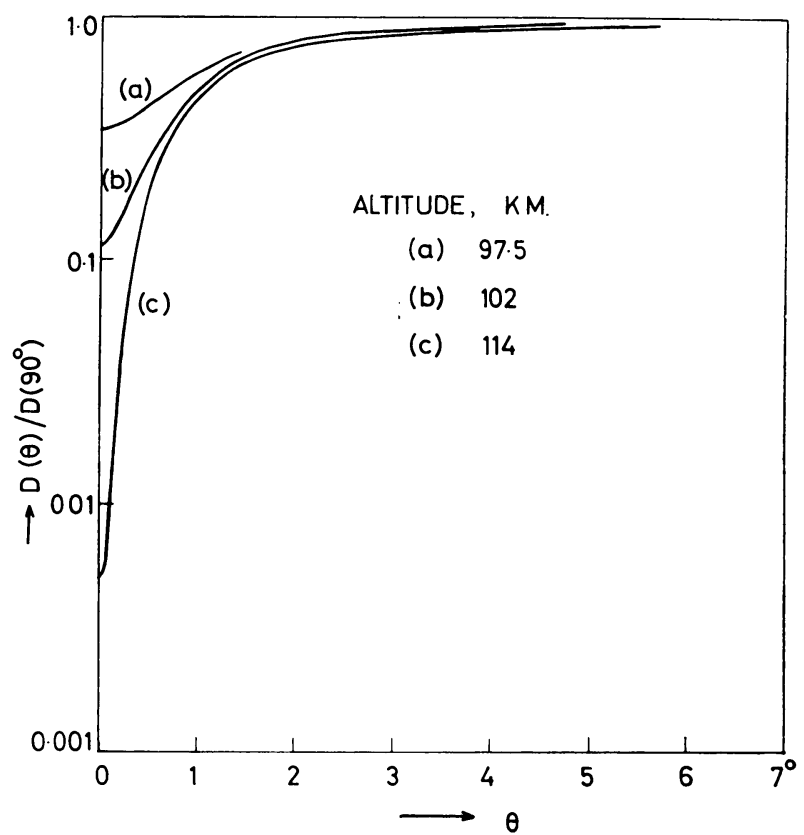

FIG. 2. $D(\theta)=$ coefficient describing diffusion normal to a plane slab of ionization at angle $\theta$ to the magnetic field.

is still proportional to the reciprocal of atmospheric density (except for trains which are closely field-aligned). In the transition region the effective coefficient will thus increase more slowly than reciprocal density and if one attempts to fit an exponential height profile it will lead to an apparently increased scale height.

Some striking experimental results which support the views expressed above have been reported by Watkins (1965). He used a $500 \mathrm{MHz}$ radar with an aerial half-power beam width of $1.6^{\circ}$ and found that both the rate and duration of meteor echoes increased by a considerable factor when the aerial beam was less than a degree or so from normality with the field lines. At the wavelength used, the finite velocity of the meteor combined with a finite diffusion rate normally causes considerable reduction in echo amplitude (the finite initial radius of the train is an additional factor). Watkins has shown, however, that if $D$ were reduced by between one and two orders of magnitude the finite velocity effect would be greatly reduced and both echo amplitude and duration would be increased sufficiently to explain his results. Inspection of Figure 2 shows that this is easily satisfied for field aligned trains at altitudes above $98 \mathrm{~km}$. Since the minimum value of $\theta$ for the trains observed is equal to the deviation of the aerial beam from normality with the field, it is clear from Figure 2 that we require the latter angle to be less than $1^{\circ}$ for a significant effect, in good agreement with the experimental result of Watkins. 


\section{Reference}

Watkins, C.D. (1965) Nature, 208, 1027.

\section{DISCUSSION}

Elford: Radio observations made at Adelaide, South Australia, indicate that the rate of change of $D$ with height for Geminid shower trains is much closer to the height variation predicted by theory than for any other shower or sporadic meteors. This suggests that the mode of formation of the train also affects the rate of diffusion.

Whipple: What do you expect will be the effect of heavy negative ions on meteor-cloud diffusion? Will there be enough negative ions to count?

Kaiser: Electron attachment in meteor trains becomes important at altitudes below about $90 \mathrm{~km}$. The presence of negative ions will modify the diffusion slightly, but this is likely to occur only at altitudes below that at which the Earth's magnetic field exerts an influence. 